\title{
Research Article \\ Similarity Solutions to Nonlinear Diffusion/Harry Dym Fractional Equations
}

\author{
Chao Yue $(\mathbb{D}$, Guijuan Liu, Kun Li, and Hanhui Dong \\ College of Medical Information Engineering, Shandong First Medical University \& Shandong Academy of Medical Sciences, \\ Tai'an 271000, China \\ Correspondence should be addressed to Chao Yue; yuechao_71@163.com
}

Received 17 October 2020; Revised 29 January 2021; Accepted 9 February 2021; Published 23 February 2021

Academic Editor: Sergey Shmarev

Copyright (c) 2021 Chao Yue et al. This is an open access article distributed under the Creative Commons Attribution License, which permits unrestricted use, distribution, and reproduction in any medium, provided the original work is properly cited.

By using scalar similarity transformation, nonlinear model of time-fractional diffusion/Harry Dym equation is transformed to corresponding ordinary fractional differential equations, from which a travelling-wave similarity solution of time-fractional Harry Dym equation is presented. Furthermore, numerical solutions of time-fractional diffusion equation are discussed. Again, through another similarity transformation, nonlinear model of space-fractional diffusion/Harry Dym equation is turned into corresponding ordinary differential equations, whose two similarity solutions are also worked out.

\section{Introduction}

Nonlinear partial differential equations arise in many fields of engineering, physics, and applied mathematics. During the last few decades, nonlinear fractional partial differential equations have gained much attention due to their applications in many branches of science and engineering such as porous media, fluid flow, fractals, heat conduction, control theory, dynamical processes, and other areas. It is generally known that fractional calculus can propose better results than classical calculus. Many methods have been used to study and analyze fractional differential equations, in which the Lie-group analysis method is an effective tool to investigate symmetries of ordinary and partial differential equations. Later, this method was generalized to study fractional partial differential equations [1-9]. Djordjevic and Atanackovic obtained similarity solutions to nonlinear fractional heat conduction equation and Burgers/KdV equations [9]. It is very critical to mention two recent papers [10,11]. First, in Ref. [10], the authors presented and discussed a fractional nonlinear partial differential equation by use of similarity reductions and recovered some interesting results associated with Harry Dym-type equations. In addition, in Ref. [11], the fractional nonlinear space-time wave-diffusion equation was discussed and solved by the similarity method utilizing frac- tional derivatives in the Caputo, Riesz-Feller, and Riesz senses. In this work, we shall treat a nonlinear model of time-fractional diffusion/Harry Dym equation

$$
\frac{\partial^{\alpha} u}{\partial t^{\alpha}}=u^{n} \frac{\partial^{n} u}{\partial x^{n}}, 0<\alpha<1, n=2,3
$$

and further study nonlinear model of space-fractional diffusion/Harry Dym equation

$$
\frac{\partial u}{\partial t}=u^{\beta} \frac{\partial^{\beta} u}{\partial x^{\beta}}, 2 \leq \beta \leq 3,
$$

with $2 \leq \beta \leq 3$, we can obtain all equations between the diffusion and Harry Dym equation. In the following, we want to study similarity solutions with Equation (1), including a travelling-wave similarity solution and a kind of numerical solutions. Furthermore, two similarity solutions in Equation (2) are also produced.

First of all, we recall several associated notations. For continuous function $f(t)$, the left Riemann-Liouville fractional 
derivative of order $0<\alpha<1$ is given as

$$
\begin{aligned}
\frac{d^{\alpha} f}{d t^{\alpha}} & =\frac{1}{\Gamma(1-\alpha)} \frac{d}{d t} \int_{0}^{t} \frac{f(\tau)}{(t-\tau)^{\alpha}} d \tau \\
& =\frac{1}{\Gamma(1-\alpha)}\left[\frac{f(0)}{t^{\alpha}}+\int_{0}^{t} \frac{f^{(1)}(\tau)}{(t-\tau)^{\alpha}} d \tau\right],
\end{aligned}
$$

where $\Gamma($.$) is the Euler Gamma function$

$$
\Gamma(\alpha)=\int_{0}^{\infty} e^{-z} z^{\alpha-1} d z
$$

Similarly, for $n-1<\beta<n$, we have

$$
\frac{d^{\beta} f}{d t^{\beta}}=\frac{1}{\Gamma(n-\beta)} \frac{d^{n}}{d t^{n}} \int_{0}^{t} \frac{f(\tau)}{(t-\tau)^{\beta+1-n}} d \tau .
$$

\section{Travelling-Wave Similarity Solution of Time- Fractional Harry Dym Equation}

Firstly, we will prove that nonlinear model (1) possesses similarity solutions, consider Lie-group scaling transformation by introducing new variables $\tilde{t}, \tilde{x}, \tilde{u}$ in the form [9]

$$
t=\lambda \tilde{t}, x=\lambda^{p} \tilde{x}, u=\lambda^{q} \tilde{u}(\tilde{x}, \tilde{t}),
$$

where $p$ and $q$ are parameters to be determined later. It is easy to verify that the transformed equation reads

$$
\lambda^{q-\alpha} \frac{\partial^{\alpha} \tilde{u}}{\partial \tilde{t}^{\alpha}}=\lambda^{(n+1) q-n p} \tilde{u}^{n} \frac{\partial^{n} \tilde{u}}{\partial \tilde{x}^{n}}
$$

which implies that $q-\alpha=(n+1) q-n p$, that is

$$
p-q=\frac{\alpha}{n}
$$

Since

$$
\frac{t}{\tilde{\tilde{t}}}=\left(\frac{x}{\tilde{x}}\right)^{\frac{1}{p}}, \frac{u}{\tilde{u}}=\left(\frac{x}{\tilde{x}}\right)^{\frac{q}{p}}
$$

in order to obtain the travelling-wave similarity solution to time-fractional Harry Dym equation

$$
\frac{\partial^{\alpha} u}{\partial t^{\alpha}}=u^{3} u_{x x x}, 0<\alpha<1
$$

we consider the similarity transformation

$$
u=x^{\frac{q}{p}} U(\xi), \xi=\frac{c t}{x}-1,
$$

where $p, q$, and $c$ are constants to be determined. We find that

$$
\frac{\partial^{\alpha} u}{\partial t^{\alpha}}=\frac{1}{\Gamma(1-\alpha)} \frac{\partial}{\partial t} \int_{0}^{t} \frac{x^{q / p} U(c t / x-1)}{(t-\tau)^{\alpha}} d \tau .
$$

Set $y=c t / x-1$; then $t-\tau=(x / c)(\xi-y), \partial / \partial t=(x / c)(d /$ $d \xi)$, and then Equation (12) becomes

$$
\begin{aligned}
& \frac{\partial^{\alpha} u}{\partial t^{\alpha}}=\frac{1}{\Gamma(1-\alpha)} \frac{c}{x} \frac{d}{d \xi} \int_{-1}^{\xi} \frac{x^{q / p} U(y)}{(x / c)^{\alpha}(\xi-y)^{\alpha}} \frac{x}{c} d y=c^{\alpha} x^{\frac{q-\alpha p}{p}} \frac{d^{\alpha} U(\xi)}{d \xi^{\alpha}}, \\
& \frac{\partial u}{\partial x}=x^{\frac{q-p}{p}}\left[\frac{q}{p} U(\xi)-(\xi+1) U^{\prime}(\xi)\right], \\
& \frac{\partial^{2} u}{\partial x^{2}}=x^{\frac{q-2 p}{p}}\left[\frac{q(q-p)}{p^{2}} U(\xi)+\frac{2(p-q)}{p}(\xi+1) U^{\prime}(\xi)+(\xi+1)^{2} U^{\prime \prime}(\xi)\right],
\end{aligned}
$$

$$
\begin{aligned}
\frac{\partial^{3} u}{\partial x^{3}}= & x^{\frac{q-3 p}{p}}\left[\frac{q(q-p)(q-2 p)}{p^{3}} U(\xi)+\frac{3(q-p)(2 p-q)}{p^{2}}\right. \\
& \left.\cdot(\xi+1) U^{\prime}(\xi)+\frac{3 q-6 p}{p}(\xi+1)^{2} U^{\prime \prime}(\xi)-(\xi+1)^{3} U^{\prime \prime \prime}(\xi)\right]
\end{aligned}
$$

Inserting (13) and (16) into Equation (10) leads to

$$
c^{\alpha} x^{\frac{q-\alpha p}{p}} \frac{d^{\alpha} U(\xi)}{d \xi^{\alpha}}=x^{\frac{4 q-3 p}{p}} T(\xi) U^{3}(\xi),
$$

It is easy to find that $q-\alpha p=4 q-3 p$, that is

$$
\frac{q}{p}=1-\frac{\alpha}{3}
$$

Then, the corresponding ordinary nonlinear fractional Harry Dym equation reads

$$
c^{\alpha} \frac{d^{\alpha} U(\xi)}{d \xi^{\alpha}}=T(\xi) U^{3}(\xi) .
$$

We take special solutions of (19) in the forms:

$$
U(\xi)=\left\{\begin{array}{l}
N \xi^{\rho}, \xi \geq 0 \\
0, \xi<0
\end{array}\right.
$$

Substituting (20) into Equation (19) gives

$$
\begin{aligned}
& \frac{c^{\alpha} N \Gamma(1+\rho)}{\Gamma(2-\alpha+\rho)}(1-\alpha+\rho) \xi^{\rho-\alpha}=\left[\frac{q(q-p)(q-2 p)}{p^{3}} \xi^{3}\right. \\
& \quad+\frac{3(q-p)(2 p-q)}{p^{2}}(\xi+1) \rho \xi^{2}+\frac{3 q-6 p}{p}(\xi+1)^{2} \rho(\rho-1) \xi \\
& \left.\quad-(\xi+1)^{3} \rho(\rho-1)(\rho-2)\right] N^{4} \xi^{4 \rho-3}
\end{aligned}
$$

which leads to $\rho-\alpha=4 \rho-3$, then

$$
\rho=1-\frac{\alpha}{3} .
$$


By using (18) and (22), from Equation (21), we get

$$
N=\left[\frac{18(3-2 \alpha) c^{\alpha} \Gamma(2-1 / 3 \alpha)}{\left(\alpha^{3}-9 \alpha\right) \Gamma(3-4 / 3 \alpha)}\right]^{\frac{1}{3}}
$$

Hence, we obtain the travelling-wave similarity solutions to Equation (10) as follows:

$$
\begin{aligned}
u(x, t) & =x^{\frac{q}{p}} N \xi^{\rho}=N(c t-x)^{1-\frac{1}{3} \alpha} \\
& =\left[\frac{18(3-2 \alpha) c^{\alpha} \Gamma(2-1 / 3 \alpha)}{\left(\alpha^{3}-9 \alpha\right) \Gamma(3-4 / 3 \alpha)}\right]^{\frac{1}{3}}(c t-x)^{1-\frac{1}{3} \alpha}
\end{aligned}
$$

From (23), we get

$$
c=\left[\frac{N^{3}\left(\alpha^{3}-9 \alpha\right) \Gamma(3-4 / 3 \alpha)}{18(3-2 \alpha) \Gamma(2-1 / 3 \alpha)}\right]^{\frac{1}{\alpha}} .
$$

Inserting (25) into (24), we finally obtain

$u(x, t)=x^{\frac{q}{p}} N \xi^{\rho}=N\left\{\left[\frac{N^{3}\left(\alpha^{3}-9 \alpha\right) \Gamma(3-4 / 3 \alpha)}{18(3-2 \alpha) \Gamma(2-1 / 3 \alpha)}\right]^{\frac{1}{\alpha}} t-x\right\}^{1-\frac{1}{3} \alpha}$

\section{Numerical Solutions of Time-Fractional Diffusion Equation}

In order to obtain the numerical solutions of time-fractional diffusion equation

$$
\frac{\partial^{\alpha} u}{\partial t^{\alpha}}=u^{2} u_{x x}, 0<\alpha<1
$$

we consider the similarity transformation

$$
u=x^{\frac{q}{p}} U(\xi), \xi=x^{-\frac{1}{p}} t
$$

where $U(\xi), \xi, p$, and $q$ are constants to be determined. We find that

$$
\begin{aligned}
\frac{\partial^{\alpha} u}{\partial t^{\alpha}} & =\frac{1}{\Gamma(1-\alpha)} x^{-\frac{1}{p}} \frac{d}{d \xi} \int_{0}^{\xi} \frac{x^{q / p} U(\tau) x^{-\alpha / p}}{(\xi-\tau)^{\alpha}} x^{\frac{1}{p}} d \tau=x^{\frac{q-\alpha}{p}} \frac{d^{\alpha} U(\xi)}{d \xi^{\alpha}} \\
\frac{\partial u}{\partial x} & =x^{\frac{q-p}{p}}\left[\frac{q}{p} U(\xi)-\frac{1}{p} \xi U^{\prime}(\xi)\right], \\
\frac{\partial^{2} u}{\partial x^{2}} & =x^{\frac{q-2 p}{p}}\left[\frac{q(q-p)}{p^{2}} U(\xi)+\frac{p-2 q+1}{p^{2}} \xi U^{\prime}(\xi)+\frac{1}{p^{2}} \xi^{2} U^{\prime \prime}(\xi)\right] .
\end{aligned}
$$

Substituting (29) and (31) into (27), we have corresponding ordinary nonlinear fractional diffusion equation

$$
\begin{aligned}
\frac{d^{\alpha} U(\xi)}{d \xi^{\alpha}}= & \frac{q(q-p)}{p^{2}} U^{3}(\xi)+\frac{p-2 q+1}{p^{2}} \xi U^{2}(\xi) U^{\prime}(\xi) \\
& +\frac{1}{p^{2}} \xi^{2} U^{2}(\xi) U^{\prime \prime}(\xi), p-q=\frac{\alpha}{2} .
\end{aligned}
$$

In what follows, we discuss its numerical solutions. In Ref. [12], suppose that a given function $f(t)$ has continuous first and second derivatives, then we get

$$
\begin{aligned}
f^{\alpha}(t)= & \frac{t^{1-\alpha}}{\Gamma(2-\alpha)}\left[f^{(1)}(t)+\sum_{n=1}^{\infty} \frac{\Gamma(n-1+\alpha)}{\Gamma(\alpha-1) n ! t^{n}} \int_{0}^{t} \tau^{n} f^{(2)}(\tau) d \tau\right] \\
& +\frac{f(0)}{t^{\alpha} \Gamma(1-\alpha)}
\end{aligned}
$$

Utilizing the integration by parts on the right-hand side gives

$$
\begin{aligned}
f^{\alpha}(t)= & \frac{1}{\Gamma(2-\alpha)}\left\{f^{(1)}(t)\left[1+\sum_{n=1}^{\infty} \frac{\Gamma(n-1+\alpha)}{\Gamma(\alpha-1) n !}\right] t^{1-\alpha}\right. \\
& \left.-\left[\frac{\alpha-1}{t^{\alpha}} f(t)+\sum_{n=2}^{\infty} \frac{\Gamma(n-1+\alpha)}{\Gamma(\alpha-1)(n-1) !}\left(\frac{f(t)}{t^{\alpha}}+\frac{\widetilde{V_{n}}}{t^{n-1+\alpha}}\right)\right]\right\} \\
& +\frac{f(0)}{t^{\alpha} \Gamma(1-\alpha)},
\end{aligned}
$$

where the moments $\widetilde{V_{n}}$ reads

$$
\widetilde{V_{n}}=(1-n) \int_{0}^{t} \tau^{n-2} f(\tau) d \tau, n=2,3, \cdots
$$

As an application, we take finite number of terms in sums of (34), that is, we take $n=2,3, \cdots, N$ with suitable chosen $N(N=7)$. Thus, from following formula for the fractional derivative, we have

$$
\begin{aligned}
f^{\alpha}(t)= & \frac{1}{\Gamma(2-\alpha)}\left\{f^{(1)}(t)\left[1+\sum_{n=1}^{N} \frac{\Gamma(n-1+\alpha)}{\Gamma(\alpha-1) n !}\right] t^{1-\alpha}\right. \\
& \left.-\left[\frac{\alpha-1}{t^{\alpha}} f(t)+\sum_{n=2}^{N} \frac{\Gamma(n-1+\alpha)}{\Gamma(\alpha-1)(n-1) !}\left(\frac{f(t)}{t^{\alpha}}+\frac{\widetilde{V_{n}}}{t^{n-1+\alpha}}\right)\right]\right\} \\
& +\frac{f(0)}{t^{\alpha} \Gamma(1-\alpha)},
\end{aligned}
$$

where $\widetilde{V_{n}}$ is given by (35). Similar to the method proposed in Refs. [13-15], the fractional equation can be replaced by a system of first-order equations of integer order by using (36). 
In what follows, we consider Equation (32). We utilize the substitution $x_{1}=U, x_{2}=U^{(1)}$ and (35) to express the fractional derivative $U^{(\alpha)}$. Then, we have the following system of first-order equations (with $\xi=t$ )

$x_{1}^{(1)}=x_{2}$,

$$
\begin{aligned}
x_{2}^{(1)}= & \frac{p^{2}}{x_{1}^{2} t^{2} \Gamma(2-\alpha)}\left\{x_{2}(t)\left[1+\sum_{n=1}^{N} \frac{\Gamma(n-1+\alpha)}{\Gamma(\alpha-1) n !}\right] t^{1-\alpha}\right. \\
& \left.-\left[\frac{\alpha-1}{t^{\alpha}} x_{1}(t)+\sum_{n=2}^{N} \frac{\Gamma(n-1+\alpha)}{\Gamma(\alpha-1)(n-1) !}\left(\frac{x_{1}(t)}{t^{\alpha}}+\frac{\widetilde{V_{n}}}{t^{n-1+\alpha}}\right)\right]\right\} \\
& +\frac{\alpha+1-p}{t} x_{2}(t)-\frac{\alpha^{2}-2 \alpha p}{4 t^{2}} x_{1}(t),
\end{aligned}
$$

with differential equations for variables $\widetilde{V}_{n}, n=2, \cdots, 7$

$\widetilde{V}_{2}^{(1)}(t)=-x_{1}(t), \widetilde{V}_{3}^{(1)}(t)=-2 t x_{1}(t), \cdots=\cdots, \widetilde{V}_{7}^{(1)}(t)=-6 t^{5} x_{1}(t)$,

subject to

$$
x_{1}(0)=x_{0}, x_{2}(0)=v_{0}, \widetilde{V_{2}}(0)=0, \widetilde{V}_{3}(0)=0, \cdots
$$

\section{Two Similarity Solutions of Space-Fractional Diffusion/Harry Dym Equation (2)}

Similarity transformation of space-fractional diffusion/Harry Dym Equation (2) is similar to the corresponding discussion of time-fractional diffusion/Harry Dym Equation (1). Take the following transformation:

$$
t=\lambda \tilde{t}, x=\lambda^{p} \tilde{x}, u=\lambda^{q} \tilde{u}(\tilde{x}, \tilde{t}) .
$$

Equation (2) is transformed to

$$
\lambda^{q-1} \frac{\partial \tilde{u}}{\partial \tilde{t}}=\lambda^{q+\beta q-\beta p} \tilde{u}^{\beta} \frac{\partial^{\beta} \tilde{u}}{\partial \tilde{x}^{\beta}}
$$

which gives that

$$
p-q=\frac{1}{\beta}
$$

In terms of

$$
\frac{x}{\tilde{\tilde{x}}}=\left(\begin{array}{l}
t \\
\overline{\tilde{t}}
\end{array}\right)^{\frac{1}{p}}, \frac{u}{\tilde{u}}=\left(\begin{array}{l}
t \\
\tilde{\tilde{t}}
\end{array}\right)^{q},
$$

we have

$$
\xi=t^{-p} x, u=t^{q} U(\xi)
$$

Then, it is easy to find

$$
u_{t}=t^{q-1}\left[q U(\xi)-p \xi U^{\prime}(\xi)\right],
$$

forn $-1<\beta<n$, by use of definition (5), one can compute that

$$
\frac{d^{\beta} u(x, t)}{d x^{\beta}}=\frac{1}{\Gamma(n-\beta)} \frac{\partial^{n}}{\partial x^{n}} \int_{0}^{x} \frac{u(\tilde{x}, t)}{(x-\tilde{x})^{\beta+1-n}} d \tilde{x} .
$$

Let

$$
I(x, t)=\frac{1}{\Gamma(n-\beta)} \int_{0}^{x} \frac{u(\tilde{x}, t)}{(x-\tilde{x})^{\beta+1-n}} d \tilde{x} .
$$

we have

$$
\frac{\partial^{n} I(x, t)}{\partial x^{n}}=\frac{1}{\Gamma(n-\beta)} \frac{\partial^{n}}{\partial x^{n}} \int_{0}^{x} \frac{t^{q} U\left(t^{-p} \tilde{x}\right)}{(x-\tilde{x})^{\beta+1-n}} d \tilde{x} .
$$

Set $\zeta=t^{-p} \tilde{x}$; then, we have

$$
x-\tilde{x}=t^{p}(\xi-\zeta), \frac{\partial^{n}}{\partial x^{n}}=t^{-n p} \frac{d^{n}}{d \xi^{n}} .
$$

Substituting the above calculations into Equation (46), we have

$$
\begin{aligned}
\frac{d^{\beta} u(x, t)}{d x^{\beta}} & =\frac{1}{\Gamma(n-\beta)} t^{-n p} \frac{d^{n}}{d \xi^{n}} \int_{0}^{\xi} \frac{t^{q} U(\zeta) t^{p}}{t^{p(\beta+1-n)}(\xi-\zeta)^{\beta+1-n}} d \zeta \\
& =t^{q-\beta p} \frac{d^{\beta} U(\xi)}{d \xi^{\beta}} .
\end{aligned}
$$

Inserting the above results into (2) yields

$$
t^{q-1}\left[q U(\xi)-p \xi U^{\prime}(\xi)\right]=t^{q+\beta q-\beta p} U^{\beta}(\xi) \frac{d^{\beta} U(\xi)}{d \xi^{\beta}},
$$

which leads to

$$
p-q=\frac{1}{\beta},
$$

which is equivalent to (42). Thus, we have the following fractional ordinary differential system:

$$
q U(\xi)-p \xi U^{\prime}(\xi)=U^{\beta}(\xi) \frac{d^{\beta} U(\xi)}{d \xi^{\beta}} .
$$

Let us consider the solution in this form:

$$
U(\xi)=W \xi^{\sigma}
$$


and then insert (54) into (53); we have

$$
(q-p \sigma) \xi^{\sigma}=W^{\beta} \frac{\Gamma(1+\sigma)}{\Gamma(2+\sigma-\beta)}(1-\beta+\sigma) \xi^{\sigma+\sigma \beta-\beta}
$$

which gives $\sigma=\sigma+\sigma \beta-\beta$, that is

$$
\sigma=1
$$

In terms of (52) and (56), from Equation (55), we get

$$
W=\left[\frac{\Gamma(3-\beta)}{\beta(\beta-2)}\right]^{\frac{1}{\beta}} \text {. }
$$

Then, we get the following similarity solutions to Equation (2):

$$
u(x, t)=\left[\frac{\Gamma(3-\beta)}{\beta(\beta-2)}\right]^{\frac{1}{\beta}} x t^{-\frac{1}{\beta}} .
$$

Similar to the discussion of the time-fractional Harry Dym Equation (10), we can obtain the travelling-wave similarity solution to space-fractional diffusion/Harry Dym Equation (2). To obtain this solution a suitable similarity transformation reads

$$
u=t^{\frac{q}{p}} U(\xi), \xi=\frac{x}{t}-\bar{c},
$$

where $p, q$, and $c$ are constants to be determined later. We see that

$$
\begin{aligned}
\frac{\partial u}{\partial t} & =t^{\frac{q-p}{p}}\left[\frac{q}{p} U(\xi)-(\xi+\bar{c}) U^{\prime}(\xi)\right], \\
\frac{\partial^{\beta} u}{\partial x^{\beta}} & =\frac{1}{\Gamma(n-\beta)} \frac{\partial^{n}}{\partial x^{n}} \int_{0}^{x} \frac{t^{q / p} U(\bar{x} / t-\bar{c})}{(x-\bar{x})^{\beta+1-n}} d \bar{x} .
\end{aligned}
$$

Set $z=\bar{x} / t-\bar{c}$, and then $x-\bar{x}=t(\xi-z), \partial^{n} / \partial x^{n}=t^{-n}\left(d^{n}\right.$ $\left./ d \xi^{n}\right)$; then, Equation (61) becomes

$$
\frac{\partial^{\beta} u}{\partial x^{\beta}}=\frac{1}{\Gamma(n-\beta)} t^{-n} \frac{d^{n}}{d \xi^{n}} \int_{-1}^{\xi} \frac{t^{q / p} U(z)}{t^{\beta+1-n}(\xi-z)^{\beta+1-n}} t d z=t^{\frac{q-\beta p}{p}} \frac{d^{\beta} U(\xi)}{d \xi^{\beta}} .
$$

Inserting (60) and (62) into (2) yields

$$
t^{\frac{q-p}{p}}\left[\frac{q}{p} U(\xi)-(\xi+\bar{c}) U^{\prime}(\xi)\right]=t^{\frac{q+\beta q-\beta p}{p}} U^{\beta}(\xi) \frac{d^{\beta} U(\xi)}{d \xi^{\beta}}
$$

which gives rise to

$$
\frac{q}{p}=1-\frac{1}{\beta}
$$

Thus, we get the following fractional ordinary differential system:

$$
\frac{d^{\beta} U(\xi)}{d \xi^{\beta}}=\left(1-\frac{1}{\beta}\right) U^{1-\beta}(\xi)-(\xi+\bar{c}) U^{-\beta}(\xi) U^{\prime}(\xi)
$$

We take special solutions of (65) in the forms

$$
U(\xi)=\left\{\begin{array}{l}
U_{1} \xi^{\delta}, \xi \geq 0 \\
0, \xi<0
\end{array}\right.
$$

Substituting (66) into Equation (65) gives

$$
\frac{U_{1} \Gamma(1+\delta)}{\Gamma(2+\delta-\beta)}(1-\beta+\delta) \xi^{\delta-\beta}=U_{1}^{1-\beta}\left[\left(1-\frac{1}{\beta}\right) \xi-\delta(\xi+\bar{c})\right] \xi^{\delta-\delta \beta-1}
$$

which implies

$$
\delta=1-\frac{1}{\beta}
$$

Then, we have

$$
U_{1}=\left[\frac{\bar{c} \Gamma(3-\beta-1 / \beta)}{(\beta-1) \Gamma(2-1 / \beta)}\right]^{\frac{1}{\beta}}
$$

Thus, we obtain the following travelling-wave similarity solution:

$$
u(x, t)=\left[\frac{\bar{c} \Gamma(3-\beta-1 / \beta)}{(\beta-1) \Gamma(2-1 / \beta)}\right]^{\frac{1}{\beta}}(x-\bar{c} t)^{1-\frac{1}{\beta}} .
$$

\section{Conclusions}

In this paper, through similarity transformations, two nonlinear models of time-fractional and space-fractional diffusion/Harry Dym equation are transformed to corresponding ordinary fractional differential equations, from which four similarity solutions are presented, including travelling-wave similarity solutions and numerical solutions. The technology presented in this paper can be applied to other fractional partial differential equations, such as multi-equation systems and $(2+1)$-dimensional equations, to get more various similarity solutions, which will enrich and supplement the known results.

\section{Data Availability}

No data were used to support this study.

\section{Conflicts of Interest}

The authors declare that they have no conflicts of interest. 


\section{Acknowledgments}

This work was supported by the Natural Science Foundation of China (Grant Nos. 11971475, 11805114), the Natural Science Foundation of Shandong Province (Grant Nos. ZR2016AL04, ZR2016FL05, and ZR2017MF039), and the High-Level Training Project of Taishan Medical University (No. 2015GCC07).

\section{References}

[1] R. Bagley and P. Torvik, "A theoretical basis for the application of fractional calculus to viscoelasticity," Journal of Rheology, vol. 27, pp. 201-210, 1983.

[2] A. Carpinteri and F. Mainardi, Fractals and Fractional Calculus in Continuum Mechanics, Springer, Wien, 1997.

[3] R. K. Gazizov, A. A. Kasatkin, and S. Y. Lukashchuk, "Symmetry properties of fractioanl diffusion equation," Physica Scripta, vol. 2009, no. T136, pp. 014016-014020, 2009.

[4] Q. Huang and R. Zhdanov, "Symmetries and exact solutions of the time fractional Harry Dym equation with RiemannLiouville derivative," Physica A: Statistical Mechanics and its Applications, vol. 409, pp. 110-118, 2014.

[5] Q. Huang and S. F. Shen, "Lie symmetries and group classification of a class of time fractional evolution systems," Journal of Mathematical Physics, vol. 56, no. 12, pp. 123504-123514, 2015.

[6] G. W. Wang and T. Z. Xu, "Invariant analysis and explicit solutions of the time fractional nonlinear perturbed Burgers equation," Nonlinear Analysis: Modelling and Control, vol. 20, pp. 570-584, 2015.

[7] H. Z. Liu, "Complete group classifications and symmetry reductions of the fractional fifth-order KdV types of equations," Studies in Applied Mathematics, vol. 131, no. 4, pp. 317-330, 2013.

[8] Y. F. Zhang, J. Q. Mei, and X. Z. Zhang, "Symmetry properties and explicit solutions of some nonlinear differential and fractional equations," Applied Mathematics and Computation, vol. 337, pp. 408-418, 2018.

[9] V. D. Djordjevic and T. M. Atanackovic, "Similarity solutions to nonlinear heat conduction and Burgers/Korteweg-de Vries fractional equations," Journal of Computational and Applied Mathematics, vol. 222, no. 2, pp. 701-714, 2008.

[10] F. S. Costa, J. C. A. Soares, A. R. G. Plata, and E. C. de Oliveira, "On the fractional Harry Dym equation," Computational and Applied Mathematics, vol. 37, pp. 2862-2876, 2018.

[11] F. S. Costa, J. A. P. F. Marão, J. C. A. Soares, and E. C. de Oliveira, "Similarity solution to fractional nonlinear space-time diffusionwave equation," Journal of Mathematical Physics, vol. 56, article 033507, 2015.

[12] T. M. Atanackovic and B. Stankovic, "An expansion formula for fractional derivatives and its applications," Fractional Calculus and Applied Analysis, vol. 7, no. 3, pp. 365-378, 2004.

[13] T. M. Atanackovic and B. Stankovic, "On a numerical scheme for solving differential equations of fractional order," Mechanics Research Communications, vol. 35, no. 7, pp. 429-438, 2008.
[14] L. Yuan and O. P. Agrawal, "A numerical scheme for dynamic systems containing fractional derivatives," in Proceedings of DETC'98 1998 ASME Design Engineering Technical Conferences, Atlanta Georgia, 1998.

[15] L. Yuan and O. P. Agrawal, "A numerical scheme for dynamic systems containing fractional derivatives," Journal of Vibration and Acoustics, vol. 124, no. 2, pp. 321-324, 2002. 